\title{
HECKE ALGEBRAS AND AFFINE FLAG VARIETIES IN CHARACTERISTIC $p$
}

\author{
TOBIAS SCHMIDT
}

\begin{abstract}
Let $G$ be a split semi-simple $p$-adic group and let $\mathscr{H}$ be its Iwahori-Hecke algebra with coefficients in the algebraic closure $\overline{\mathbb{F}}_{p}$ of $\mathbb{F}_{p}$. Let $\mathcal{F}$ be the affine flag variety over $\overline{\mathbb{F}}_{p}$ associated with $G$. We show, in the simply connected simple case, that a torusequivariant $K^{\prime}$-theory of $\mathcal{F}$ (with coefficients in $\overline{\mathbb{F}}_{p}$ ) admits an action of $\mathscr{H}$ by Demazure operators and that this provides a model for the regular representation of $\mathscr{H}$.
\end{abstract}

\section{Contents}

1. Introduction

2. Algebraic loop groups and their affine flag varieties

3. Equivariant $K$-theory

3.1. Schubert classes

3.2. Demazure operators

3.3. Iwahori-Hecke algebras

References

\section{INTRODUCTION}

Let $F$ be a $p$-adic local field with ring of integers $o_{F}$ and let $\overline{\mathbb{F}}_{p}$ be the algebraic closure of its residue field. Let $G$ be a connected reductive linear algebraic group over $F$ and denote by $G(F)$ the group of its $F$-rational points. For a choice of Iwahori subgroup $I \subset G(F)$, the corresponding Iwahori-Hecke algebra $\mathscr{H}_{\mathbb{\mathbb { F }}_{p}}$ over $\overline{\mathbb{F}}_{p}$ equals the convolution algebra $\overline{\mathbb{F}}_{p}[I \backslash G(F) / I]$ of $\overline{\mathbb{F}}_{p}$-valued functions with finite support on the double cosets of $G(F) \bmod I$. The algebra $\mathscr{H}_{\mathbb{\mathbb { F }}_{p}}$ and a certain extension $\mathscr{H}_{\overline{\mathbb{F}}_{p}}^{(1)}$ of it associated to the maximal pro- $p$ subgroup of $I$ - the pro- $p$ Iwahori-Hecke algebra - were systematically studied by Vignéras [23], [24, [25]. Among their first applications is the mod $p$ local Langlands program for the group $G(F)[6]$.

In this note we only deal with $\mathscr{H}_{\overline{\mathbb{F}}_{p}}$ and realize it as an equivariant algebraic $K$-theory of its affine flag variety $\mathcal{F}$ over $\overline{\mathbb{F}}_{p}$. We view this result as a first step to analyze the mod

The author would like to acknowledge support of the Heisenberg programme of Deutsche Forschungsgemeinschaft (SCHM 3062/1-1). 
$p$ geometry of the algebras $\mathscr{H}_{\overline{\mathbb{F}}_{p}}$ and $\mathscr{H}_{\overline{\mathbb{F}}_{p}}^{(1)}$. In particular, we hope that the geometry of affine Springer fibres in $\mathcal{F}$ [5] will eventually shed a light on the representation theory of these algebras, in analogy to the classical case of the finite complex flag variety [11. We also note that the occurence of affine geometry in the mod $p$ setting can be viewed as a consequence of the failure of the Bernstein presentation for the algebras $\mathscr{H}_{\mathbb{F}_{p}}$ and $\mathscr{H}_{\overline{\mathbb{F}}_{p}}^{(1)}$ [24].

To give more details about the present article, let us assume that $G$ extends to a split simply connected semisimple and simple group scheme over the integers. We denote its base change to $\overline{\mathbb{F}}_{p}$ by the same letter. Let $T \subset G$ be a maximal torus. We choose an Iwahori subgroup $\mathcal{B} \subset G\left(\overline{\mathbb{F}}_{p}[[t]]\right)$ whose projection to $G\left(\overline{\mathbb{F}}_{p}\right)$ contains $T$ and let

$$
\mathcal{G}=G\left(\overline{\mathbb{F}}_{p}((t))\right) / G\left(\overline{\mathbb{F}}_{p}[[t]]\right) \quad \text { resp. } \quad \mathcal{F}=G\left(\overline{\mathbb{F}}_{p}((t))\right) / \mathcal{B}
$$

be the affine Grassmannian resp. the affine flag variety of $G$ over $\overline{\mathbb{F}}_{p}$. They are ind-schemes whose structure can be defined through the increasing union of Schubert varieties $S_{w}$ indexed by elements $w$ in the algebraic cocharacters $\Lambda$ of $T$ resp. in the affine Weyl group $W$ of $G$. By definition, $S_{w}$ equals the closure of the $\mathcal{B}$-orbit through $w *$ and has a natural structure as finite dimensional projective variety over $\overline{\mathbb{F}}_{p}$. Let $\tilde{T}$ be the extension of $T$ by the 'turning torus' $\mathbf{G}_{m}$ (which corresponds to the derivation element $d$ in the Kac-Moody setting). Then $\tilde{T}$ acts on $\mathcal{G}$ and $\mathcal{F}$ and preserves the stratification into Schubert varieties. Denote by $K_{\tilde{T}}^{\prime}\left(S_{w}\right)$ the Grothendieck group of $\tilde{T}$-equivariant coherent module sheaves on $S_{w}$ and put $\mathbb{K}_{\tilde{T}}^{\prime}(\cdot):=K_{\tilde{T}}^{\prime}(\cdot) \otimes \overline{\mathbb{F}}_{p}$. We define

$$
\mathbb{K}_{\tilde{T}}^{\prime}(\mathcal{F}):=\underset{w}{\lim } \mathbb{K}_{\tilde{T}}^{\prime}\left(S_{w}\right)
$$

and show that the Schubert classes $\left[\mathcal{O}_{S_{w}}\right]$ provide an $\overline{\mathbb{F}}_{p}[\tilde{T}]$-basis for $\mathbb{K}_{\tilde{T}}^{\prime}(\mathcal{F})$. Here, $\overline{\mathbb{F}}_{p}[\tilde{T}]$ denotes the ring of algebraic functions on $\tilde{T}$ over $\overline{\mathbb{F}}_{p}$. The analogous result holds for $\mathcal{G}$. Letting $\pi: \mathcal{F} \rightarrow \mathcal{G}$ denote the projection, the pull-back

$$
\pi^{*}: \mathbb{K}_{\tilde{T}}^{\prime}(\mathcal{G}) \hookrightarrow \mathbb{K}_{\tilde{T}}^{\prime}(\mathcal{F})
$$

is a linear injection whose image can be described explicitly. To each simple affine reflection $s$ corresponds a smooth Bruhat-Tits group scheme $P_{s}$ over $\overline{\mathbb{F}}_{p}[[t]]$ with connected fibres and with corresponding partial affine flag variety $\mathcal{F}_{s}$. The projection $\pi_{s}: \mathcal{F} \rightarrow \mathcal{F}_{s}$ is a $\mathbf{P}^{1}$-bundle. One obtains a Demazure operator $D_{s}:=\pi_{s}^{*} \pi_{s *}$ on $\mathbb{K}_{\tilde{T}}^{\prime}(\mathcal{F})$ in analogy to the classical case of the finite flag variety treated in [3]. The operators $D_{s}$ satisfy braid relations and quadratic relations which are exactly the ones appearing in the IwahoriMatsumoto presentation of $\mathscr{H}_{\mathbb{F}_{p}}$. The latter is described in terms of the $\overline{\mathbb{F}}_{p}$-basis given by the characteristic functions $\tau_{w}$ of the double cosets $I w I$ in the classical way [9], however in our situation, the parameters satisfy $q_{s}=0$ for all $s$, since $\overline{\mathbb{F}}_{p}$ has characteristic $p$. Letting $\tau_{s}$ act through $D_{s}$ therefore turns $\mathbb{K}_{\tilde{T}}^{\prime}(\mathcal{F})$ into a right module over $\mathscr{H}_{\mathbb{F}_{p}[\tilde{T}]}=\mathscr{H}_{\mathbb{F}_{p}} \otimes \overline{\mathbb{F}}_{p}[\tilde{T}]$. 
In this situation, our main result says that mapping the function $(-1)^{\ell(w)} \tau_{w}$ to the Schubert class $\left[\mathcal{O}_{S_{w}}\right]$ for each $w$ induces an isomorphism

$$
\mathscr{H}_{\mathbb{F}_{p}[\tilde{T}]} \stackrel{\simeq}{\longrightarrow} \mathbb{K}_{\tilde{T}}^{\prime}(\mathcal{F})
$$

as right $\mathscr{H}_{\overline{\mathbb{F}}_{p}[\tilde{T}]}$-modules. Here, $\ell$ denotes the length function on $W$. Finally, specialising the isomorphism at the unit element $\tilde{T}=1$ yields an isomorphism between $\mathscr{H}_{\mathbb{F}_{p}}$ and the absolute $K^{\prime}$-theory $\mathbb{K}^{\prime}(\mathcal{F})$ (with coefficients in $\overline{\mathbb{F}}_{p}$ ) as right $\mathscr{H}_{\mathbb{F}_{p}}$-modules. In particular, any irreducible right $\mathscr{H}_{\mathbb{\mathbb { F }}_{p}}$-module appears as a quotient of $\mathbb{K}^{\prime}(\mathcal{F})$. As a corollary, we show that the restriction of $\pi^{*}$ to the module spanned by Schubert classes supported on anti-dominant cocharacters $\Lambda_{-}$realizes the regular representation for the spherical Hecke algebra $\overline{\mathbb{F}}_{p}\left[G\left(o_{F}\right) \backslash G(F) / G\left(o_{F}\right)\right]$ viewed inside $\mathscr{H}_{\overline{\mathbb{F}}_{p}}$ via the $\bmod p$ Satake isomorphism [7]. We remark that all our results hold in fact with $\overline{\mathbb{F}}_{p}$ replaced by any subfield $k \subseteq \overline{\mathbb{F}}_{p}$.

We expect all results to extend to arbitrary connected and split reductive groups. Moreover, replacing the Iwahori-subgroup $\mathcal{B} \subset L G$ by the inverse image of the unipotent radical

of the Borel subgroup of $G$ in question should extend all results from $\mathscr{H}_{\mathbb{F}_{p}}$ to $\mathscr{H}_{\overline{\mathbb{F}}_{p}}^{(1)}$. We leave these issues for future work.

To make a brief comparison with the complex case, we recall that over the complex numbers the link between representations of the Iwahori-Hecke algebra and equivariant $K$-theory was discovered by Lusztig [14 and occupies a central position in applications to the local Langlands program. We only mention the proof of the Deligne-Langlands conjecture by Kazhdan-Lusztig [11] where it is shown that the equivariant $K$-homology of the complex Steinberg variety of triples affords a natural Hecke action through $q$-analogues of Demazure operators and that this provides a model for the regular representation of the Hecke algebra. We also point out that the theory of Demazure operators on equivariant $K$-theory of affine flag manifolds is well-established in the complex Kac-Moody setting, due to the work of Kostant-Kumar [12] and Kashiwara-Shimozono [10].

Acknowledgements: The author would like to thank Elmar Große-Klönne and Marc Levine for some interesting conversations related to this topic.

\section{Algebraic LOOP GRoups AND their AFFine Flag VARIETIES}

We recall some definitions and concepts from [4], [17]. Let $k$ be a field and let $K=k((t))$ be the field of formal Laurent series over $k$. Let $G$ be a simply connected semi-simple and simple group over $k$ which is split over $k$. Let $L G$ be its associated algebraic loop group, i.e. the functor

$$
R \mapsto L G(R)=G(R((t)))
$$

on the category of $k$-algebras. It is representable by an ind-scheme over $k$. Let $L^{+} G$ be its associated positive loop group which is an affine group scheme over $k$ representing the 
functor

$$
R \mapsto L^{+} G(R)=G(R[[t]]) .
$$

Let $\mathcal{B}$ be the inverse image under the morphism pr: $L^{+} G \rightarrow G, t \mapsto 0$ of a Borel subgroup in $G$. Thus, $\mathcal{B}$ equals a closed subscheme of an infinite dimensional affine $k$-space. The affine Grassmannian $\mathcal{G}$ resp. the affine flag variety $\mathcal{F}$ of $L G$ is the ind-scheme over $k$

$$
\mathcal{G}=L G / L^{+} G \quad \text { resp. } \quad \mathcal{F}=L G / \mathcal{B}
$$

representing the functor $R \mapsto L G(R) / L^{+} G(R)$ resp. $R \mapsto L G(R) / \mathcal{B}(R)$. The natural projection map $\pi: \mathcal{F} \rightarrow \mathcal{G}$ is a proper and Zariski locally trivial fibration with fibers $L^{+} G / \mathcal{B} \simeq G / \operatorname{pr}(\mathcal{B})$ (the split case in [17, 8.e.1]). The group $L G$ acts on $\mathcal{G}$ and $\mathcal{F}$ by left translations. Evaluating $G$ on the structure map $R \mapsto R((t))$ gives an inclusion $G \subset L G$. In this way, $G$ and all its subgroups act on $\mathcal{G}$ and $\mathcal{F}$.

For example, let $G=\mathrm{SL}_{d}$, the special linear group. Then $L G$ equals the union of subfunctors $L_{n} G$ where $L_{n} G(R)$ is the set of matrices $M=\sum_{i \geqslant-n} M_{i} t^{i}$ of determinant 1 with $M_{i}$ a $d \times d$-matrix with entries in $R$. Each $L_{n} G$ is representable as a closed subscheme of the affine scheme $\prod_{i \geqslant-n} \mathbf{A}_{k}^{d^{2}}$ and the inclusion $L_{n} G \subset L_{n+1} G$ is a closed embedding. This defines the ind-structure on $L G$. To make the ind-structure of $\mathcal{G}$ and $\mathcal{F}$ explicit, recall that a lattice $\mathscr{L} \subset R((t))^{d}$ is a $R[[t]]$-submodule of $R((t))^{d}$ such that there is $n \geqslant 0$ with

$$
t^{n} R[[t]]^{d} \subseteq \mathscr{L} \subseteq t^{-n} R[[t]]^{d}
$$

and such that the quotient $t^{-n} R[[t]]^{d} / \mathscr{L}$ is a locally free $R$-module of finite rank. If $\bigwedge^{d} \mathscr{L}=R[[t]]$, the lattice is called special. We denote the set of special lattices over $R$ by $\mathcal{L}(R)$ and those for fixed $n$ by $\mathcal{L}_{n}(R)$. Then $\mathcal{L}_{n}$ is representable by a projective $k$-scheme. Indeed, the morphism

$$
\mathcal{L}_{n}(R) \rightarrow \operatorname{Grass}_{n d}\left(t^{-n} k[[t]]^{d} / t^{n} k[[t]]^{d}\right)(R), \mathscr{L} \mapsto \mathscr{L} / t^{n} R[[t]]^{d}
$$

defines a closed embedding into the Grassmannian of $n d$-dimensional subspaces of the $2 n d$ dimensional $k$-vector space $t^{-n} k[[t]]^{d} / t^{n} k[[t]]^{d}$. Note that multiplication by $1+t$ induces a (unipotent) automorphism on the latter vector space which induces an automorphism of the Grassmannian. The image of $\mathcal{L}_{n}$ equals its fixed point variety and hence, is indeed closed. Each element of $L G(R)$ gives rise to a special lattice in $R((t))^{d}$ by applying it to the standard lattice $R[[t]]^{d}$. Since $G(R[[t]])$ equals the stabilizer of the standard lattice, we obtain an isomorphism $\mathcal{G}(R) \simeq \mathcal{L}(R)$. This defines the ind-structure of $\mathcal{G}$ in the case of $\mathrm{SL}_{d}$. Now a lattice chain in $R((t))^{d}$ is a chain

$$
\mathscr{L}_{0} \supset \mathscr{L}_{1} \supset \cdots \supset \mathscr{L}_{d-1} \supset t \mathscr{L}_{0}
$$

with lattices $\mathscr{L}_{i}$ in $R((t))^{d}$ such that each quotient $\mathscr{L}_{i} / \mathscr{L}_{i+1}$ is a locally free $R$-module of rank 1 . Each element of $L G(R)$ gives rise to a lattice chain with $\mathscr{L}_{0}$ special by applying it to the standard lattice chain

$$
\mathscr{L}_{i}:=\bigoplus_{j=1, \ldots, d-i} R[[t]] e_{j} \oplus \underset{j=d-i+1, \ldots, d}{\bigoplus_{i}} t R[[t]] e_{j}
$$


where $e_{1}, \ldots, e_{d}$ denotes the standard basis of $R((t))^{d}$. Since $\mathcal{B}(R)$ equals the stabilizer of the standard lattice chain, we obtain an isomorphism between $\mathcal{F}(R)$ and the space of lattice chains $\mathscr{L}_{\bullet}$ in $R((t))^{d}$ such that $\mathscr{L}_{0}$ is special. In this optic, the projection $\pi$ is given by sending a chain $\mathscr{L}_{\bullet}$ to $\mathscr{L}_{0}$ and, hence, is a fiber bundle whose fibres are all isomorphic to the usual flag variety $G / \operatorname{pr}(\mathcal{B})$ of $G$. This defines the ind-structure of $\mathcal{F}$ in the case of $\mathrm{SL}_{d}$. For more details we refer to [5].

In the following, we are forced to work with a less explicit realization of the ind-structure on $\mathcal{F}$. To define it requires more notation. Our assumptions on $G$ imply that $\mathcal{B}(k)$ is an Iwahori-subgroup of $G(K)$ and hence, defines a fundamental chamber in the Bruhat-Tits building of $G(K)$. We denote by $B$ the associated smooth group scheme with connected fibres over $k[[t]]$. Then $\mathcal{B}=L^{+} B$ in the notation of [17, 1.b]. Similarly, to any wall $F_{i}$ (i.e. facet of codimension 1) of this chamber, we let $P_{i}$ be the smooth group scheme with connected fibres over $k[[t]]$ such that $P_{i}(k[[t]])$ is equal to the parahoric subgroup of $G(K)$ associated to $F_{i}$. To $P_{i}$ corresponds an infinite-dimensional affine group scheme $L^{+} P_{i}$ over $k$ with $L^{+} P_{i}(R)=P_{i}(R[[t]])$. The fpqc-quotient $\mathcal{F}_{i}:=L G / L^{+} P_{i}$ is representable by an ind-scheme over $k$, the partial affine flag variety associated to $F_{i}$. The projection morphism

$$
\pi_{i}: \mathcal{F} \rightarrow \mathcal{F}_{i}
$$

is a principal $L^{+} P_{i} / L^{+} B$-bundle for the Zariski topology (the split case in [17, Thm. 1.4]). Moreover, $L^{+} P_{i} / L^{+} B \simeq \mathbf{P}_{k}^{1}$, the projective line over $k$, according to [17, Prop. 8.7]. We denote the reflection in the wall $F_{i}$ by $s_{i}$.

Let $T$ be a maximal torus in $G$ contained in the Borel subgroup $\operatorname{pr}(\mathcal{B})$ and let $N_{G}(T)$ be its normalizer. Let $W_{0}=N_{G}(T) / T$ be the finite Weyl group of $G$ with longest element $w_{0}$. It may be viewed as the group generated by reflections in $\mathcal{S}_{0}:=\left\{s_{1}, \ldots, s_{l}\right\}$. Let $\Phi=\Phi(G, T)$ be the root system and let $\Phi^{+}$be the set of positive roots distinguished by $\operatorname{pr}(\mathcal{B})$. Let $\Lambda:=X_{*}(T)$ denote the group of cocharacters of $T$. We view $\Lambda$ as a subgroup of $T(k[[t]])$ via the mapping $\lambda \mapsto \lambda(t)$. In particular, $\Lambda$ acts on $\mathcal{G}$ and $\mathcal{F}$.

Let $W$ be the affine Weyl group of $G$. The group $W$ is an affine Coxeter group with set of generators $\mathcal{S}:=\mathcal{S}_{0} \cup\left\{s_{0}\right\}$. We denote by $\leqslant$ the Bruhat-Chevalley (partial) order on $W$ and by $\ell(\cdot)$ the length function. Since $G$ is simply connected, $\Lambda$ coincides with the coroot lattice whence $W$ may be written as the semi-direct product $W=\Lambda \rtimes W_{0}$. When considering $\Lambda$ as a subgroup of $W$ (whose group law is written multiplicatively), we will use exponential notation.

Let $P$ be either $B$ or $P_{i}$ or $G$ and write for a moment $\mathcal{F}_{P}=L G / L^{+} P$. Denote by * $=L^{+} P \in \mathcal{F}_{P}$ the base point. Let $W^{P}$ be the set of representatives in $W$ of minimal length for the right cosets $W / W_{P}$ where $W_{P} \subset W$ equals the subgroup generated by 1 or $s_{i}$ or $\mathcal{S}_{0}$. The $(B, P)$-Schubert cell $C_{w}=C_{w}(B, P)$ is the reduced subscheme

$$
L^{+} B w * \subset \mathcal{F}_{P}
$$


The $(B, P)$-Schubert variety $S_{w}=S_{w}(B, P)$ is the reduced subscheme with underlying set the Zariski closure of $C_{w}$ in $L G / L^{+} P$. It is a projective variety over $k$. Given $u \leqslant w$ there is a closed immersion $S_{u} \hookrightarrow S_{w}$ and

$$
L G / L^{+} P=\bigcup_{w \in W^{P}} S_{w}
$$

defines the ind-structure on $\mathcal{F}_{P}$. If we let $\mathcal{F}_{P}^{n}$ be the (finite) union over the $S_{w}$ with $\ell(w) \leqslant n$, then $\mathcal{F}_{P}=\cup_{n} \mathcal{F}_{P}^{n}$ defines the ind-structure on $\mathcal{F}_{P}$, too. In case of $P=P_{i}$, we shall write $\mathcal{F}_{i}=\cup_{n} \mathcal{F}_{i}^{n}$. In the following, we sometimes drop the base point $*$ from the notation.

Let $\Delta=\left\{\alpha_{1}, \ldots, \alpha_{l}\right\}$ be the set of simple roots in $\Phi$ associated with $\mathcal{S}_{0}$ and let $\theta$ be the highest root. Denote by $\Phi^{\text {aff }}$ the collection of affine roots $(\alpha, m)$ with $\alpha \in \Phi$ and $m \in \mathbb{Z}$. Then $\alpha_{0}:=(-\theta, 1)$ is the remaining affine simple root which induces the reflection $s_{0}$. For each affine root $(\alpha, m)$, there is an associated inclusion

$$
\phi_{(\alpha, m)}: S L_{2} \hookrightarrow L G
$$

defined as follows [17, (9.8)]. The vector part $\alpha$ defines a $k$-morphism $S L_{2} \hookrightarrow G$ in the usual way which induces by functoriality $L\left(S L_{2}\right) \hookrightarrow L G$. The map $\phi_{(\alpha, m)}$ equals the composition with the morphism

$$
S L_{2} \longrightarrow L\left(S L_{2}\right),\left(\begin{array}{ll}
a & b \\
c & d
\end{array}\right) \mapsto\left(\begin{array}{cc}
a & b t^{m} \\
c t^{-m} & d
\end{array}\right)
$$

The image of $\phi_{\alpha_{i}}$ is contained in a Levi subgroup of the parabolic $L^{+} P_{i} \subset L G$ for $i=$ $0, \ldots, l$. There is an irreducible $k$-linear representation $V_{i}$ of the group scheme $L^{+} P_{i}$ whose pull-back via $\phi_{\alpha_{i}}$ equals the unique irreducible representation of $S L_{2}$ of dimension 2. Let $L G \times{ }^{L^{+}} P_{i} \mathbf{P}\left(V_{i}\right)$ be the associated projective bundle over $\mathcal{F}_{i}$. Let $v_{i}$ be a highest weight vector in $V_{i}$ and for $g \in L G$ denote by $\left[g, v_{i}\right]$ the class of $\left(g, k v_{i}\right) \in L G \times{ }^{L^{+}} P_{i} \mathbf{P}\left(V_{i}\right)$. We then have a morphism of $\mathbf{P}^{1}$-bundles $\mathcal{F} \rightarrow L G \times{ }^{L^{+} P_{i}} \mathbf{P}\left(V_{i}\right)$ given by $g L^{+} B \mapsto\left[g, v_{i}\right]$.

Lemma 2.2. The morphism $\mathcal{F} \stackrel{\simeq}{\longrightarrow} L G \times{ }^{L^{+} P_{i}} \mathbf{P}\left(V_{i}\right)$ is an isomorphism.

Proof. It suffices to check that the morphism is bijective on fibres [13, IV.4.2.E(3)]. By $L G$-equivariance we may check this over the base point. However, the stabilizer of $v_{i}$ in $L^{+} P_{i}$ equals $L^{+} B$ and since $V_{i}$ is irreducible, this implies $L^{+} P_{i} / L^{+} B \simeq \mathbf{P}\left(V_{i}\right)$.

In [4, p. 54] Faltings constructs a central extension

$$
1 \longrightarrow \mathbf{G}_{m} \longrightarrow \bar{L} G \stackrel{\psi}{\longrightarrow} L G \longrightarrow 1
$$

which acts on all line bundles on $\mathcal{F}$ (denoted $\tilde{L} G$ in loc.cit.). The copy of $\mathbf{G}_{m}$ corresponds to the central element $c$ in the Kac-Moody setting. We consider $T$ as a subgroup of $L G$ and consider its inverse image $\bar{T}$ in $\bar{L} G$, i.e. $\bar{T}:=\psi^{-1}(T)$. The central extension has a unique splitting over $L^{+} B$ and we regard $T$ as being contained in $\bar{T}$. There is a fundamental character $\rho_{i} \in X^{*}(\bar{T})$ whose restriction to $T$ gives the character of $V_{i}$. 
On the other hand, there is a natural identification

$$
L G \times{ }^{L^{+} P_{i}} \mathbf{P}\left(V_{i}\right) \simeq \mathbf{P}\left(L G \times{ }^{L^{+} P_{i}} V_{i}\right)
$$

and we have the family of line bundles $\mathcal{O}(n)$ for $n \in \mathbb{Z}$ on this space. Each character $\lambda \in X^{*}(\bar{T})$ yields an associated line bundle $\mathcal{L}_{\lambda}=L G \times^{L^{+} B} k_{\lambda}$ on $\mathcal{F}$ [17, 10.a.]. Here, $k_{\lambda}$ denotes the one-dimensional $\bar{T}$-representation induced by $\lambda$.

Lemma 2.3. Let $0 \leqslant i \leqslant l$. The pull-back of $\mathcal{O}(-1)$ to $\mathcal{F}$ equals $\mathcal{L}_{\rho_{i}}$. The $\mathcal{L}_{\rho_{i}}$ form a basis of the Picard group $\operatorname{Pic}(\mathcal{F})$ of $\mathcal{F}$.

Proof. Consider the two-dimensional $L^{+} P_{i}$-representation $V_{i}$ of weight $\rho_{i}$ and highest weight vector $v_{i} \in V_{i}$ as above. Consider the line bundle $\mathcal{L}_{\rho_{i}}=G \times{ }^{L^{+} B} k v_{i}$ on $\mathcal{F}$ and fix a point $g L^{+} B \in \mathcal{F}$. The fibre of $\mathcal{L}_{\rho_{i}}$ in $g L^{+} B$ equals $\left[g, v_{i}\right]$. Since the isomorphism $\mathcal{F} \simeq \mathbf{P}\left(G \times{ }^{L^{+} P_{i}} V_{i}\right)$ takes $g L^{+} B$ to $\left[g, v_{i}\right]$, we see that $\mathcal{L}_{\rho_{i}}$ equals the pull-back of the tautological line bundle $\mathcal{O}(-1)$. The second assertion follows from this together with [17, Prop. 10.1].

We need to introduce yet another torus. Let $P$ be either $B$ or $P_{i}$ or $G$ and abbreviate $\mathcal{F}_{P}=L G / L^{+} P$. For a given $k$-algebra $R$, its group of units $a \in R^{\times}$acts on the rings $R[[t]]$ and $R((t))$ via $t^{m} \mapsto a^{m} t^{m}$ ('turning the loop'). This induces an action of $\mathbf{G}_{m}$ on $L G$ preserving the subgroup $L^{+} P$. We obtain an action on $\mathcal{F}_{P}$ which commutes with the action of $T$. We write

$$
\tilde{T}:=\mathbf{G}_{m} \times T
$$

for the extended torus. This copy of $\mathbf{G}_{m}$ corresponds to the derivation element $d$ in the Kac-Moody setting. The action of the extended torus preserves the Schubert cell $C_{w}$. Indeed, we may write $w=w^{\prime} e^{\lambda}$ with $w^{\prime} \in W_{0}$ and $\lambda \in \Lambda$. Given $a \in R^{\times}$one has

$$
a(w *)=a\left(w^{\prime}\right) a(\lambda(t)) *=w^{\prime} \lambda(a t) *=w^{\prime} \lambda(a) \lambda(t) *=\lambda(a) w *=w *
$$

since $\lambda(a) \in T(R)$. Therefore $\tilde{T}$ indeed stabilizes $C_{w}$. It induces an action of $\tilde{T}$ on the Schubert variety $S_{w}$. Even more, $w *$ is the only fixpoint in $C_{w}$ for the action of $T$ and $\tilde{T}$. Namely, the cell $C_{w}$ is isomorphic to a finite direct sum over copies of 'root spaces' $\mathbf{A}_{k}^{1}$ for $\tilde{T}$ indexed by affine roots $(\alpha, m)$ and with 'root vectors' $t^{m} X_{\alpha}$. The isomorphism is $\tilde{T}$-equivariant and maps $w *$ to the origin in $\oplus \mathbf{A}_{k}^{1}$. Here, $X_{\alpha}$ denotes a root vector for the root group $U_{\alpha} \subset G$ and the torus $\tilde{T}$ acts on $t^{m} X_{\alpha}$ through the character $(a, s) \mapsto a^{m} \alpha(s)$, e.g. [4, p. 46/53].

\section{EqUiVARIANT $K$-THEORY}

3.1. Schubert classes. Let $P$ be either $B$ or $P_{i}$ or $G$ and let $\mathcal{F}_{P}=L G / L^{+} P$. Suppose for a moment that $S \subset \mathcal{F}_{P}$ is a $\tilde{T}$-stable ind-subvariety which is, in fact, a finite-dimensional algebraic $k$-variety. We then have the abelian category of $\tilde{T}$-equivariant coherent modules on $S$. We denote the corresponding Grothendieck group by $K_{\tilde{T}}^{\prime}(S)$. Let $f: Y \rightarrow S$ 
be an equivariant morphism to $S$ from another $\tilde{T}$-variety $Y$. If $f$ is flat, then pullback of modules induces a homomorphism $f^{*}: K_{\tilde{T}}^{\prime}(S) \rightarrow K_{\tilde{T}}^{\prime}(Y)$. If $f$ is proper, there is a push-forward homomorphism $f_{*}: K_{\tilde{T}}^{\prime}(Y) \rightarrow K_{\tilde{T}}^{\prime}(S)$ induced by $\sum_{j}(-1)^{j} R^{j} f_{*}$ [19, 1.10/11]. Note that if $f$ is actually a closed embedding, then of course $R^{j} f_{*}=0$ for $j>0$. Applying this to the structure map of $S$, the tensor product endowes the group $K_{\tilde{T}}^{\prime}(S)$ with a module structure for the group ring

$$
\mathbb{Z}[\tilde{T}]:=\mathbb{Z}\left[X^{*}(\tilde{T})\right]=K_{\tilde{T}}^{\prime}(\operatorname{Spec} k) .
$$

Let $H \subseteq \tilde{T}$ be a closed subgroup. Restricting the $\tilde{T}$-action to $H$ induces a linear homomorphism

$$
\mathbb{Z}[H] \otimes_{\mathbb{Z}[\tilde{T}]} K_{\tilde{T}}^{\prime}(\mathcal{F}) \longrightarrow K_{H}^{\prime}(\mathcal{F})
$$

It is often bijective [15].

We define the Grothendieck group of $\mathcal{F}_{P}$ to be

$$
K_{\tilde{T}}^{\prime}\left(\mathcal{F}_{P}\right):=\underset{n}{\lim _{\longrightarrow}} K_{\tilde{T}}^{\prime}\left(\mathcal{F}_{P}^{n}\right)=\underset{w \in W^{P}}{\lim _{\longrightarrow}} K_{\tilde{T}}^{\prime}\left(S_{w}\right)
$$

in analogy with the case of the complex Kashiwara affine flag manifold $(k=\mathbb{C})$ treated in [22, 2.2.1]. The transition maps here are induced by the push-forward along the $\tilde{T}$ equivariant closed embeddings $\mathcal{F}_{P}^{n} \hookrightarrow \mathcal{F}_{P}^{n+1}$ resp. $S_{u} \hookrightarrow S_{w}$ for $u \leqslant w$.

Let $U_{P}^{n}:=\mathcal{F}_{P}^{n} \backslash \mathcal{F}_{P}^{n-1}$ with $U_{P}^{0}=$ Spec $k$. We let $j_{n}: U_{P}^{n} \hookrightarrow \mathcal{F}_{P}^{n}$ denote the corresponding open embedding.

Lemma 3.2. There is a linear isomorphism $K_{\tilde{T}}^{\prime}\left(U_{P}^{n}\right) \simeq \oplus_{\ell(w)=n} \mathbb{Z}[\tilde{T}]$.

Proof. We have $U_{P}^{n}=\coprod_{\ell(w)=n} C_{w}$ with a linear $\tilde{T}$-action on $C_{w} \simeq \mathbf{A}_{k}^{\ell(w)}$, cf. discussion at the end of previous section. So the claim follows from homotopy invariance of $K_{\tilde{T}}^{\prime}$ 20, $4.2]$.

We have the element $\left[\mathcal{O}_{S_{w}}\right]$ in $K_{\tilde{T}}^{\prime}\left(\mathcal{F}_{P}\right)$ for $w \in W^{P}$. In the complex Kac-Moody setting these Schubert classes give rise to ' $\mathbb{Z}$-bases' in the corresponding $K$-theories of the affine flag manifold [10], [12]. In the following, we are rather interested in certain fields of positive characteristic $p>0$ where we are not aware of corresponding facts in the literature. The following results have straightforward proofs and are sufficient for our purposes. We let

$$
\mathbb{K}_{H}^{\prime}(\cdot):=K_{H}^{\prime}(\cdot) \otimes_{\mathbb{Z}} k \text { and } k[\cdot]:=\mathbb{Z}[\cdot] \otimes_{\mathbb{Z}} k
$$

for any closed subgroup $H \subseteq \tilde{T}$. If $H=1$, we write $\mathbb{K}^{\prime}$ instead of $\mathbb{K}_{\{1\}}^{\prime}$. We denote by $\overline{\mathbb{F}}_{p}$ the algebraic closure of the finite field $\mathbb{F}_{p}$ with $p$ elements.

Proposition 3.3. Let $k \subseteq \overline{\mathbb{F}}_{p}$. The $k[\tilde{T}]$-module $\mathbb{K}_{\tilde{T}}^{\prime}\left(\mathcal{F}_{P}^{n}\right)$ is free on the basis given by the Schubert classes $\left[\mathcal{O}_{S_{w}}\right]$ with $\ell(w)=n$. If $H \subseteq \tilde{T}$ is closed subgroup, then (3.1) is an isomorphism after tensoring with $k$. 
Proof. We have $\mathbb{Z}[H] \otimes_{\mathbb{Z}} K^{\prime} \bullet=K_{H}^{\prime}$ for those $H$-varieties which have trivial $H$-action and where $K^{\prime} \bullet$ denotes ordinary $K^{\prime}$-theory. Applying this to $\bullet=1$ and using

$$
\mathbb{K}^{1}(\operatorname{Spec} k)=k^{\times} \otimes_{\mathbb{Z}} k=0,
$$

the first equivariant $K^{\prime}$-group of $U_{P}^{n}=\coprod_{\ell(w)=n} C_{w}$ thus vanishes upon tensoring with $k$. The localization sequence, tensored with $k$,

$$
0 \longrightarrow \mathbb{K}_{H}^{\prime}\left(\mathcal{F}_{P}^{n-1}\right) \longrightarrow \mathbb{K}_{H}^{\prime}\left(\mathcal{F}_{P}^{n}\right) \stackrel{j_{n}^{*}}{\longrightarrow} \mathbb{K}_{H}^{\prime}\left(U_{P}^{n}\right) \longrightarrow 0
$$

is therefore exact [20, 2.7]. Since a preimage under $j_{n}^{*}$ of the unit vector at the $w$-th position in $\oplus \ell(w)=n \mathbb{Z}[H]$ is given by $\left[\mathcal{O}_{S_{w}}\right]$, the first assertion follows now by induction on $n$ with $H=\tilde{T}$. The bijectivity of (3.1) follows now easily from this.

Corollary 3.4. Let $k \subseteq \overline{\mathbb{F}}_{p}$. The $k[\tilde{T}]$-module $\mathbb{K}_{\tilde{T}}^{\prime}\left(\mathcal{F}_{P}\right)$ is free on the basis given by the $\left[\mathcal{O}_{S_{w}}\right]$ with $w \in W^{P}$.

Remark: The ind-structure on $\mathcal{F}_{P}$ may also be defined via the $(P, P)$-Schubert varieties equal to the closure of the $L^{+} P$-orbits in $\mathcal{F}_{P}$ [17]. Since an $L^{+} P$-orbit is not a topological cell anymore, we chose $(B, P)$-Schubert varieties to calculate the equivariant $K^{\prime}$-theory of $\mathcal{F}_{P}$.

For each $n$ the set of fixpoints $\left(\mathcal{F}_{P}^{n}\right)^{\tilde{T}}$ is a non-empty closed subvariety of $\mathcal{F}_{P}^{n}$. We let $\iota_{n}:\left(\mathcal{F}_{P}^{n}\right)^{\tilde{T}} \hookrightarrow \mathcal{F}_{n}$ be the corresponding closed embedding. We have the following simple version of the Thomason concentration theorem for fixed points varieties [21, Thm. 2.1].

Proposition 3.5. Let $k \subseteq \overline{\mathbb{F}}_{p}$. The variety $\left(\mathcal{F}_{P}^{n}\right)^{\tilde{T}}$ equals the union of the finitely many points $w * \in \mathcal{F}_{P}$ for $\ell(w) \leqslant n$ and $w \in W^{P}$. One has a linear isomorphism

$$
\iota_{n *}: \mathbb{K}_{\tilde{T}}^{\prime}\left(\left(\mathcal{F}_{P}^{n}\right)^{\tilde{T}}\right) \stackrel{\simeq}{\longrightarrow} \mathbb{K}_{\tilde{T}}^{\prime}\left(\mathcal{F}_{P}^{n}\right)
$$

which maps the class of the point $w *$ to $\left[\mathcal{O}_{S_{w}}\right]$.

Proof. One has

$$
\left(\mathcal{F}_{P}^{n}\right)^{\tilde{T}}=\coprod_{\ell(w) \leqslant n}\left(C_{w}\right)^{\tilde{T}}=\coprod_{\ell(w) \leqslant n}\{w *\}
$$

by the discussion at the end of the previous section. For the second assertion we consider the cartesian diagramm

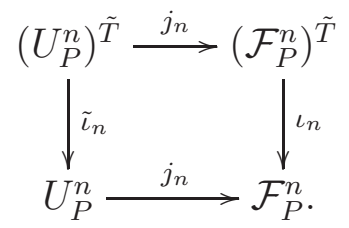

Flat equivariant base change for equivariant $K^{\prime}$-theory yields $j_{n}^{*} \iota_{n *}=\tilde{\iota}_{n *} j_{n}^{*}[19$, 1.11]. We obtain a commutative diagram 


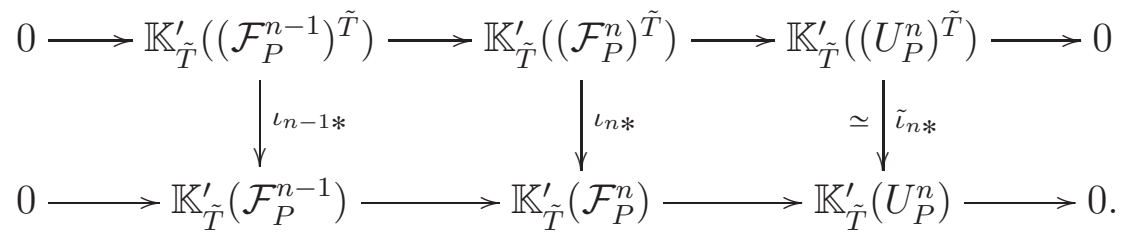

where the left-hand square commutes by functoriality for proper maps [19, 1.11] and the right-hand isomorphism comes from homotopy invariance. The lower horizontal sequence is exact by the proof of the preceding proposition and this implies the exactness of the upper horizontal sequence. The result follows now by induction on $n$.

3.2. Demazure operators. We fix a number $n$ and consider the preimage of $\mathcal{F}_{i}^{n}$ under the projection $\pi_{i}: \mathcal{F} \rightarrow \mathcal{F}_{i}$ from 2.1. Since $\pi_{i}$ is flat and proper we have the homomorphisms

$$
K_{\tilde{T}}^{\prime}\left(\pi_{i}^{-1} \mathcal{F}_{i}^{n}\right) \stackrel{\pi_{i *}}{\rightleftarrows} K_{\pi_{i}^{*}}^{\prime}\left(\mathcal{F}_{i}^{n}\right) .
$$

In analogy to the case of the finite flag variety [3] and the complex Kashiwara affine flag manifold [10], we define a linear operator on $K_{\tilde{T}}^{\prime}\left(\pi_{i}^{-1} \mathcal{F}_{i}^{n}\right)$ by

$$
D_{i}:=\pi_{i}^{*} \circ \pi_{i *}
$$

Lemma 3.6. The operator $D_{i}$ extends to an operator on $K_{\tilde{T}}^{\prime}(\mathcal{F})$.

Proof. Since $\pi_{i}$ is a principal $\mathbf{P}^{1}$-bundle, each $\pi_{i}^{-1} \mathcal{F}_{i}^{n}$ is a closed algebraic subvariety in $\mathcal{F}$ and these varieties define, for varying $n$, the ind-structure on $\mathcal{F}$. It suffices therefore to show that $D_{i}$ is compatible with the transition map $K_{\tilde{T}}^{\prime}\left(\pi_{i}^{-1} \mathcal{F}_{i}^{n-1}\right) \rightarrow K_{\tilde{T}}^{\prime}\left(\pi_{i}^{-1} \mathcal{F}_{i}^{n}\right)$. We consider the cartesian diagram

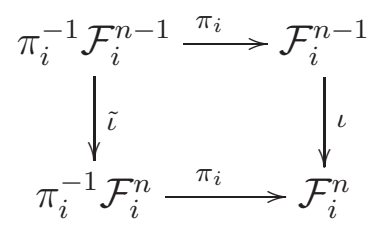

where the map $\iota$ is the natural inclusion and the map $\tilde{\iota}$ is induced by it. Flat equivariant base change for equivariant $K^{\prime}$-theory yields $\pi_{i}^{*} \iota_{*}=\tilde{\iota}_{*} \pi_{i}^{*}$ and functoriality for proper maps yields $\iota_{*} \pi_{i_{*}}=\pi_{i *} \tilde{\iota}_{*}$. Hence

$$
\tilde{\iota}_{*} \pi_{i}^{*} \pi_{i *}=\pi_{i}^{*} \iota_{*} \tilde{\pi}_{i *}=\pi_{i}^{*} \pi_{i *} \tilde{\iota}_{*} .
$$

We denote the resulting operator on $K_{\tilde{T}}^{\prime}(\mathcal{F})$ by the same symbol $D_{i}$. As in [3, Prop. 2.6], the projective bundle theorem in $K$-theory identifies the operator $D_{i}$ as an idempotent projector as follows. We note that under the isomorphism $\mathcal{F} \simeq \mathbf{P}\left(L G \times{ }^{L^{+} P_{i}} V_{i}\right)$ of 2.2 
the morphism $\pi_{i}: \mathcal{F} \rightarrow \mathcal{F}_{i}$ becomes the projective space bundle associated to the rank 2 vector bundle $L G \times{ }^{L^{+} P_{i}} V_{i} \rightarrow \mathcal{F}_{i},[g, v] \mapsto g L^{+} P_{i}$.

Lemma 3.7. Let $0 \leqslant i \leqslant l$. The correspondence

$$
\left(a_{0}, a_{1}\right) \mapsto 1 \otimes \pi_{i}^{*} a_{0}+\mathcal{L}_{\rho_{i}} \otimes \pi_{i}^{*} a_{1}
$$

induces a $\mathbb{Z}[\tilde{T}]$-linear isomorphism

$$
K_{\tilde{T}}^{\prime}\left(\mathcal{F}_{i}^{n}\right)^{\oplus 2} \stackrel{\simeq}{\longrightarrow} K_{\tilde{T}}^{\prime}\left(\pi_{i}^{-1} \mathcal{F}_{i}^{n}\right)
$$

for any $n$.

Proof. Since $\mathcal{L}_{\rho_{i}}=\mathcal{O}(-1)$ by 2.3 , we apply the (equivariant) projective bundle theorem [20, Thm. 3.1] to $\pi_{i}^{-1} \mathcal{F}_{i}^{n} \rightarrow \mathcal{F}_{i}^{n}$.

Proposition 3.8. In the situation of the lemma, the operator $D_{i}$ on $K_{\tilde{T}}^{\prime}\left(\pi_{i}^{-1} \mathcal{F}_{i}^{n}\right)$ has the property

$$
D_{i}\left(1 \otimes \pi_{i}^{*} a_{0}+\mathcal{L}_{\rho_{i}} \otimes \pi_{i}^{*} a_{1}\right)=1 \otimes \pi_{i}^{*} a_{0} .
$$

Proof. According to [18, Lemma 8.1.1(c)] the modules $M_{0}:=\pi_{i}^{*} a_{0}$ and $M_{1}(-1):=$ $\mathcal{O}(-1) \otimes M_{1}$ with $M_{1}:=\pi_{i}^{*} a_{1}$ satisfy $\pi_{i *} M_{0}=a_{0}$ and $\pi_{i *} M_{1}(-1)=0$ and, moreover, they are regular modules in the sense of Mumford, cf. loc.cit. Then [18, Lemma 8.1.3] implies $R^{j} \pi_{i *} M_{0}=R^{j} \pi_{i *} M_{1}(-1)=0$ for $j>0$.

Corollary 3.9. The operator $D_{i}$ on $K_{\tilde{T}}^{\prime}(\mathcal{F})$ satisfies $D_{i}^{2}=D_{i}$.

Remark: According to the preceding proposition, $D_{i}$ may be also be viewed as an algebraic characteristic $p$ analogue of the operator $D_{i}$ defined by Kostant-Kumar on the topological equivariant $K$-theory of the complex analytic Kac-Moody flag manifold [12, Def. (3.6)].

We compute the action of $D_{i}$ on the elements $\left[\mathcal{O}_{S_{w}}\right]$ of $K_{\tilde{T}}^{\prime}(\mathcal{F})$.

Lemma 3.10. Let $Z \subset \mathcal{F}_{i}^{n}$ be a closed $\tilde{T}$-subvariety and let $\left[\mathcal{O}_{Z}\right]$ be its class in $K_{\tilde{T}}^{\prime}\left(\mathcal{F}_{i}^{n}\right)$. Then $\pi^{*}\left[\mathcal{O}_{Z}\right]=\left[\mathcal{O}_{\pi_{i}^{-1} Z}\right]$ in $K_{\tilde{T}}^{\prime}\left(\pi_{i}^{-1} \mathcal{F}_{i}^{n}\right)$.

Proof. This follows directly from the flatness of $\pi_{i}$.

For $w \in W^{P_{i}}$ let $S_{w}^{i}$ be a Schubert variety in $\mathcal{F}_{i}$. We have $\pi_{i}^{-1} S_{w}^{i}=S_{w^{\prime}}$ where $w^{\prime} \in W$ has the property: $w^{\prime}=w$ if $w s_{i}<w$ or else $w^{\prime}=w s_{i}$. This implies that for any $w \in W$ one has

$$
\pi_{i}^{-1} \pi_{i}\left(S_{w}\right)=S_{w} \quad \text { if } \quad w s_{i}<w \quad \text { or else } \pi_{i}^{-1} \pi_{i}\left(S_{w}\right)=S_{w s_{i}}
$$

Remark: Similarly, the inverse image of $S_{w} \subset \mathcal{G}$ under $\pi$ equals $S_{w w_{0}} \subset \mathcal{F}$ where $w_{0}$ denotes the longest element in $W_{0}$. Indeed, the inverse image $\pi^{-1} S_{w}$ is a Schubert variety in $\mathcal{F}$ and $w w_{0}$ is the representative of maximal length in its coset modulo $W_{0}$. 
Lemma 3.12. Let $w \in W$ and suppose $S_{w} \subset \pi_{i}^{-1} \mathcal{F}_{i}^{n}$ for some $n$. Then

$$
\pi_{i *}\left[\mathcal{O}_{S_{w}}\right]=\left[\mathcal{O}_{\pi_{i}\left(S_{w}\right)}\right]
$$

in $K_{\tilde{T}}^{\prime}\left(\mathcal{F}_{i}^{n}\right)$.

Proof. Consider the commutative diagram

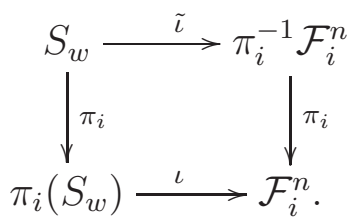

where $\iota$ and $\tilde{\iota}$ are the corresponding closed embeddings. Functoriality for proper maps implies $\pi_{i *} \tilde{\iota}_{*}=\iota_{*} \pi_{i *}$ on $K_{\tilde{T}}^{\prime}\left(S_{w}\right)$. However, $\iota_{*}$ and $\tilde{\iota}_{*}$ are exact functors on coherent modules, so that we are reduced to show:

$$
R^{j} \pi_{i *}\left(\mathcal{O}_{S_{w}}\right)=0 \text { for } j>0 \text { and } \pi_{i *}\left(\mathcal{O}_{S_{w}}\right)=\mathcal{O}_{\pi_{i}\left(S_{w}\right)} .
$$

We distinguish two cases. Assume first that $w s_{i}<w$. Then $\pi_{i}^{-1} \pi_{i}\left(S_{w}\right)=S_{w}$ by 3.11 so that our commutative diagramm is even cartesian. Since $\pi_{i}$ is a projective space bundle, our claims follow from [18, 8.1.1/8.1.3]. Assume now $w s_{i}>w$. Then $\pi_{i}^{-1} \pi_{i}\left(S_{w}\right)=S_{w s_{i}}$ by 3.11 and the map $\pi_{i}: S_{w s_{i}} \rightarrow \pi_{i}\left(S_{w}\right)$ is a proper birational morphism [2, Remark 3.2.3]. Our claims follow since $S_{w s_{i}}$ and $\pi_{i}\left(S_{w}\right)$ are normal with at most rational singularities [17, Prop. 9.6].

Proposition 3.13. Let $w \in W$. One has

$$
D_{i}\left[\mathcal{O}_{S_{w}}\right]= \begin{cases}{\left[\mathcal{O}_{S_{w}}\right]} & \text { if } w s_{i}<w \\ {\left[\mathcal{O}_{S_{w_{i}}}\right]} & \text { if } w s_{i}>w\end{cases}
$$

Proof. According to the two preceding lemmas we have $\pi_{i}^{*} \pi_{i *}\left[\mathcal{O}_{S_{w}}\right]=\left[\mathcal{O}_{\pi_{i}^{-1} \pi_{i}\left(S_{w}\right)}\right]$ so that 3.11 implies the assertion.

From now on we assume that $k \subseteq \overline{\mathbb{F}}_{p}$. We consider the endomorphism ring

$$
\operatorname{End}_{k[\tilde{T}]}\left(\mathbb{K}_{\tilde{T}}^{\prime}(\mathcal{F})\right)^{\text {op }}
$$

of $k[\tilde{T}]$-linear operators on $\mathbb{K}_{\tilde{T}}^{\prime}(\mathcal{F})$ with multiplication $(\phi \varphi)(c)=\varphi(\phi(c))$ for $c \in \mathbb{K}_{\tilde{T}}^{\prime}(\mathcal{F})$. In particular, $\mathbb{K}_{\tilde{T}}^{\prime}(\mathcal{F})$ is naturally a right module over this ring. For an element $w \in W$ with reduced decomposition $w=s_{i_{1}} \cdots s_{i_{n}}$ we define a linear operator $D_{w}$ on $\mathbb{K}_{\tilde{T}}^{\prime}(\mathcal{F})$ by

$$
D_{w}:=D_{i_{1}} \cdots D_{i_{n}}
$$

and $D_{1}:=\mathrm{id}$.

Lemma 3.14. The operator $D_{w}$ does not depend on the choice of reduced decomposition for $w$. 
Proof. According to [1, IV.§1.Prop. 5], it suffices to check for $i \neq j$ and $m_{i j}=\operatorname{ord}\left(s_{i} s_{j}\right)<$ $\infty$ the relations $D_{i} D_{j} D_{i} \cdots=D_{j} D_{i} D_{j} \cdots$ with $m_{i j}$ factors on both sides. According to 3.4 we can check this on Schubert classes. Since $W$ is crystallographic, we have $m_{i j} \in\{2,3,4,6\}$, so that the claim can be checked case-by-case. We treat the case $m_{i j}=2$, the other cases follow along the same lines, but with more notation. Since $m_{i j}=2$, we must show that $D_{i}$ and $D_{j}$ commute. So let $w \in W$ and let us argue case-by-case. First, if $w s_{i}, w s_{j}<w$, then $\left[\mathcal{O}_{S_{w}}\right]$ is a fixpoint of $D_{i} D_{j}$ and $D_{j} D_{i}$. Secondly, if $w s_{i}, w s_{j}>w$, then $w s_{i} s_{j}>w s_{j}$ and $w s_{j} s_{i}>w s_{i}$ according to case (b) in the proof of [8, Prop. 5.9] which yields

$$
\left[\mathcal{O}_{S_{w}}\right]\left(D_{i} D_{j}\right)=\left[\mathcal{O}_{S_{w s_{i}}}\right] D_{j}=\left[\mathcal{O}_{S_{w_{i} s_{j}}}\right]=\left[\mathcal{O}_{S_{w_{j} s_{i}}}\right]=\left[\mathcal{O}_{S_{w}}\right]\left(D_{j} D_{i}\right)
$$

By symmetry in $i$ and $j$, it now remains to consider the case $w s_{j}>w$ and $w s_{i}<w$. Then $\left[\mathcal{O}_{S_{w}}\right]\left(D_{i} D_{j}\right)=\left[\mathcal{O}_{S_{w_{j}}}\right]$ and $\left[\mathcal{O}_{S_{w}}\right]\left(D_{j} D_{i}\right)=\left[\mathcal{O}_{S_{w s_{j}}}\right] D_{i}$. We have either $w s_{i} s_{j}<w s_{j}$ or $w s_{i} s_{j} \leqslant w$ according to [8, Prop. 5.9]. In the first case, we are done. The second case leads via $w s_{i} s_{j} \leqslant w<w s_{j}$ to the first case.

We summarize the properties of the $D_{w}$. Let $\mathcal{S}$ be the set of generators $s_{i}$ for the affine Coxeter group $W$.

Corollary 3.15. Let $w, w^{\prime} \in W$. The linear operators $D_{w}$ on $\mathbb{K}_{\tilde{T}}^{\prime}(\mathcal{F})$ satisfy the relations

$$
\begin{aligned}
D_{w} D_{w^{\prime}} & =D_{w w^{\prime}} \quad \text { if } \ell(w)+\ell\left(w^{\prime}\right)=\ell\left(w w^{\prime}\right), \\
D_{s}^{2} & =D_{s} \quad \text { for any } s \in \mathcal{S} .
\end{aligned}
$$

Remark: Since $D_{w}$ acts linearly on $\mathbb{K}_{\tilde{T}}^{\prime}(\mathcal{F})$, there is an induced action of $D_{w}$ on

$$
\mathbb{K}_{H}^{\prime}(\mathcal{F}) \simeq k[H] \otimes_{k[\tilde{T}]} \mathbb{K}_{\tilde{T}}^{\prime}(\mathcal{F})
$$

for any closed subgroup $H \subseteq \tilde{T}$ according to 3.3 .

Remark: The projection $\pi: \mathcal{F} \rightarrow \mathcal{G}$ is a locally trivial $G / \operatorname{pr}(\mathcal{B})$-fibration and we have the linear homomorphism

$$
\pi^{*}: K_{\tilde{T}}^{\prime}\left(S_{w}\right) \rightarrow K_{\tilde{T}}^{\prime}\left(\pi^{-1} S_{w}\right)
$$

Flat equivariant base change, as in the proof of 3.6, shows that these homomorphisms commute with transition maps and give in the limit a linear homomorphism $\pi^{*}: K_{\tilde{T}}^{\prime}(\mathcal{G}) \rightarrow$ $K_{\tilde{T}}^{\prime}(\mathcal{F})$. It follows from 3.10 and the subsequent remark that $\pi^{*}$ maps the class of $S_{w} \subset \mathcal{G}$ to the class of $S_{w w_{0}} \subset \mathcal{F}$. According to 3.4, the induced morphism

$$
\pi^{*}: \mathbb{K}_{\tilde{T}}^{\prime}(\mathcal{G}) \hookrightarrow \mathbb{K}_{\tilde{T}}^{\prime}(\mathcal{F})
$$

is therefore injective. 
3.3. Iwahori-Hecke algebras. We keep the assumption $k \subseteq \overline{\mathbb{F}}_{p}$. The integral CoxeterHecke algebra of $(W, S)$ with weights $q_{s}=0$ for all $s \in \mathcal{S}$ is given as

$$
\mathscr{H}=\oplus_{w \in W} \mathbb{Z} \tilde{Y}_{w}
$$

with relations

$$
\begin{aligned}
\tilde{Y}_{w} \tilde{Y}_{w^{\prime}} & =\tilde{Y}_{w w^{\prime}} \text { if } \ell(w)+\ell\left(w^{\prime}\right)=\ell\left(w w^{\prime}\right), \\
\tilde{Y}_{s}^{2} & =-\tilde{Y}_{s} \quad \text { for any } s \in \mathcal{S},
\end{aligned}
$$

cf. [1, Ex. IV.§2.(24)]. We let $Y_{w}:=(-1)^{\ell(w)} \tilde{Y}_{w}$ for any $w$ and $\mathscr{H}_{R}:=\mathscr{H}_{\mathbb{Z}} R$ for a commutative ring $R$. We have the monoid

$$
\Lambda_{+}=\left\{\lambda \in \Lambda:\langle\lambda, \alpha\rangle \geqslant 0 \text { for all } \alpha \in \Phi^{+}\right\}
$$

of dominant cocharacters in $\Lambda$ and its corresponding monoid ring $\mathbb{Z}\left[\Lambda_{+}\right]$. One has $\ell\left(e^{\lambda}\right)=$ $\langle\lambda, 2 \rho\rangle$ for any $\lambda \in \Lambda_{+}$where $2 \rho$ equals the sum over elements from $\Phi^{+}$. Hence

$$
\Theta: \mathbb{Z}\left[\Lambda_{+}\right] \longrightarrow \mathscr{H}, \lambda \mapsto Y_{e^{\lambda}}
$$

is an injective ring homomorphism. In this way, we view $\mathbb{Z}\left[\Lambda_{+}\right]$as a subring of $\mathscr{H}$.

Proposition 3.17. Letting $Y_{w}$ act through $D_{w}$ makes $\mathbb{K}_{\tilde{T}}^{\prime}(\mathcal{F})$ a right $\mathscr{H}_{k[\tilde{T}]}$-module.

Proof. The elements $Y_{w}$ are a $\mathbb{Z}$-basis of $\mathscr{H}$ with defining relations $Y_{w} Y_{w^{\prime}}=Y_{w w^{\prime}}$ if $\ell(w)+\ell\left(w^{\prime}\right)=\ell\left(w w^{\prime}\right)$ and $Y_{s}^{2}=Y_{s}$ for any $s \in \mathcal{S}$. Acccording to 3.15 the map $Y_{w} \mapsto D_{w}$ extends therefore to a ring homomorphism between $\mathscr{H}_{k[\tilde{T}]}$ and $\operatorname{End}_{k[\tilde{T}]}\left(\mathbb{K}_{\tilde{T}}^{\prime}(\mathcal{F})\right)^{\text {op }}$.

We view the algebra $\mathscr{H}_{k[\tilde{T}]}$ via its multiplication as a right module over itself.

Theorem 3.18. There is an isomorphism of right $\mathscr{H}_{k[\tilde{T}]}$-modules

$$
\Xi: \mathscr{H}_{k[\tilde{T}]} \stackrel{\simeq}{\longrightarrow} \mathbb{K}_{\tilde{T}}^{\prime}(\mathcal{F}), Y_{w} \mapsto\left[\mathcal{O}_{S_{w}}\right] .
$$

Specialising at $\tilde{T}=1$ yields an isomorphism of right $\mathscr{H}_{k}$-modules $\mathscr{H}_{k} \simeq \mathbb{K}^{\prime}(\mathcal{F})$.

Proof. The first map is $k[\tilde{T}]$-linear and bijective [3.4. It is equivariant for the action of any $Y_{s}$ by 3.13 and therefore for the action of any $Y_{w}$. The second statement follows from the remark after 3.15 .

We show that the Grassmannian $\mathcal{G}$ gives rise to a Hecke submodule in $\mathbb{K}_{\tilde{T}}^{\prime}(\mathcal{F})$ for the image of $\Theta$. One has $\ell\left(e^{-\lambda} w\right)=\ell\left(w^{-1} e^{\lambda}\right)=\ell(w)+\ell\left(e^{-\lambda}\right)$ for any $\lambda \in \Lambda_{+}$and $w \in W_{0}$. This means that the elements from $\Lambda_{-}:=-\Lambda_{+}=w_{0}\left(\Lambda_{+}\right)$are the minimal representatives in their corresponding cosets modulo $W_{0}$. We let $\pi_{-}^{*}$ be the restriction of the homomorphism $\pi^{*}$ from (3.16) to the submodule generated by the Schubert classes indexed by $\Lambda_{-}$and consider its image $\operatorname{Im} \pi_{-}^{*} \subset \mathbb{K}_{\tilde{T}}^{\prime}(\mathcal{F})$. Note that the ring $k\left[\Lambda_{+}\right]$acts on $\mathbb{K}_{\tilde{T}}^{\prime}(\mathcal{F})$ by restricting the $\mathscr{H}_{k}$-action via $\Theta$. 
Corollary 3.19. The isomorphism $\Xi$ induces an isomorphism

$$
Y_{w_{0}} k\left[\Lambda_{+}\right] \stackrel{\simeq}{\longrightarrow} \operatorname{Im} \pi_{-}^{*}
$$

as right $k\left[\Lambda_{+}\right]$-modules. In particular, $\operatorname{Im} \pi_{-}^{*}$ is a free $k\left[\Lambda_{+}\right]$-module of rank 1 .

Proof. If $\lambda \in \Lambda_{-}$, then $e^{\lambda} w_{0}=w_{0} e^{w_{0}(\lambda)}$ with $w_{0}(\lambda) \in \Lambda_{+}$. According to 3.16 and the subsequent remarks, the $k[\tilde{T}]$-submodule $\operatorname{Im} \pi_{-}^{*}$ is therefore freely generated by the classes of the $S_{w_{0} e^{\lambda}}$ where $\lambda \in \Lambda_{+}$. Given another $\mu \in \Lambda_{+}$, one has $\ell\left(w_{0} e^{\lambda} e^{\mu}\right)=\ell\left(w_{0} e^{\lambda}\right)+\ell\left(e^{\mu}\right)$ and therefore $D_{e^{\mu}}\left[\mathcal{O}_{S_{w_{0} e^{\lambda}}}\right]=\left[\mathcal{O}_{S_{w_{0} e^{\lambda+\mu}}}\right] \in \operatorname{Im} \pi_{-}^{*}$. This implies all assertions.

Remark: Suppose the group $G$ comes from a simply-connected semi-simple and simple group scheme $G$ over $\mathbb{Z}$. Let $F$ be a non-archimedean local field, i.e. equal to a finite extension of the field $\mathbb{Q}_{p}$ of $p$-adic numbers with residue field $k$ or else equal to $k((t))$, with $k \subseteq \overline{\mathbb{F}}_{p}$ finite. Let $o_{F} \subset F$ be its ring of integers. Let $I \subset G(F)$ be an Iwahori-subgroup of the group $G(F)$. The Iwahori-Hecke algebra of $G(F)$ over $k$ equals the convolution algebra $k[I \backslash G(F) / I]$ of $k$-valued functions with finite support on the double cosets of $G(F) \bmod$ $I$. A $k$-basis is given by the characteristic functions of the double cosets $I \backslash G(F) / I$ which, in turn, are in bijection with the group $W$. Denote by $\tau_{w}$ the characteristic function of $I w I, w \in W$. By the Iwahori-Matsumoto presentation [9] there is an algebra isomorphism

$$
k[I \backslash G(F) / I] \stackrel{\simeq}{\longrightarrow} \mathscr{H}_{k}, \tau_{w} \mapsto \tilde{Y}_{w} .
$$

We thus obtain the theorem mentioned in the introduction. Let $G_{0}=G\left(o_{F}\right)$ and consider the spherical Hecke algebra $k\left[G_{0} \backslash G(F) / G_{0}\right]$. In the $p$-adic case, the mod $p$ Satake map [7] induces an algebra isomorphism

$$
k\left[G_{0} \backslash G(F) / G_{0}\right] \stackrel{\simeq}{\longrightarrow} k\left[\Lambda_{+}\right] .
$$

For the compatibilies between the various isomorphisms we refer to [16, 8C.].

\section{REFERENCES}

[1] Nicolas Bourbaki. Lie groups and Lie algebras. Chapters 4-6. Elements of Mathematics (Berlin). Springer-Verlag, Berlin, 2002. Translated from the 1968 French original by Andrew Pressley.

[2] Michel Brion and Shrawan Kumar. Frobenius splitting methods in geometry and representation theory, volume 231 of Progress in Mathematics. Birkhäuser Boston, Inc., Boston, MA, 2005.

[3] Michel Demazure. Désingularisation des variétés de Schubert généralisées. Ann. Sci. École Norm. Sup. (4), 7:53-88, 1974. Collection of articles dedicated to Henri Cartan on the occasion of his 70th birthday, I.

[4] Gerd Faltings. Algebraic loop groups and moduli spaces of bundles. J. Eur. Math. Soc. (JEMS), 5(1):41-68, 2003.

[5] Ulrich Görtz. Affine Springer fibers and affine Deligne-Lusztig varieties. In Affine flag manifolds and principal bundles, Trends Math., pages 1-50. Birkhäuser/Springer Basel AG, Basel, 2010.

[6] Elmar Grosse-Klönne. From pro- $p$ Iwahori-Hecke modules to $(\phi, \Gamma)$-modules I. Preprint 2014.

[7] Florian Herzig. A Satake isomorphism in characteristic p. Compos. Math., 147(1):263-283, 2011.

[8] James E. Humphreys. Reflection groups and Coxeter groups, volume 29 of Cambridge Studies in Advanced Mathematics. Cambridge University Press, Cambridge, 1990. 
[9] N. Iwahori and H. Matsumoto. On some Bruhat decomposition and the structure of the Hecke rings of p-adic Chevalley groups. Inst. Hautes Études Sci. Publ. Math., (25):5-48, 1965.

[10] Masaki Kashiwara and Mark Shimozono. Equivariant $K$-theory of affine flag manifolds and affine Grothendieck polynomials. Duke Math. J., 148(3):501-538, 2009.

[11] David Kazhdan and George Lusztig. Proof of the Deligne-Langlands conjecture for Hecke algebras. Invent. Math., 87(1):153-215, 1987.

[12] Bertram Kostant and Shrawan Kumar. $T$-equivariant $K$-theory of generalized flag varieties. J. Differential Geom., 32(2):549-603, 1990.

[13] Shrawan Kumar. Kac-Moody groups, their flag varieties and representation theory, volume 204 of Progress in Mathematics. Birkhäuser Boston, Inc., Boston, MA, 2002.

[14] George Lusztig. Equivariant $K$-theory and representations of Hecke algebras. Proc. Amer. Math. Soc., 94(2):337-342, 1985.

[15] Alexander S. Merkurjev. Equivariant $K$-theory. In Handbook of $K$-theory. Vol. 1, 2, pages 925-954. Springer, Berlin, 2005.

[16] Rachel Ollivier. Parabolic induction and Hecke modules in characteristic $p$ for $p$-adic $\mathrm{GL}_{n}$. Algebra Number Theory, 4(6):701-742, 2010.

[17] G. Pappas and M. Rapoport. Twisted loop groups and their affine flag varieties. Adv. Math., 219(1):118-198, 2008. With an appendix by T. Haines and Rapoport.

[18] D. Quillen. Higher algebraic K-theory. I. In Algebraic K-theory, I: Higher K-theories (Proc. Conf., Battelle Memorial Inst., Seattle, Wash., 1972), pages 85-147. Lecture Notes in Math., Vol. 341. Springer, Berlin, 1973.

[19] R. W. Thomason. Lefschetz-Riemann-Roch theorem and coherent trace formula. Invent. Math., 85(3):515-543, 1986.

[20] R. W. Thomason. Algebraic $K$-theory of group scheme actions. In Algebraic topology and algebraic K-theory (Princeton, N.J., 1983), volume 113 of Ann. of Math. Stud., pages 539-563. Princeton Univ. Press, Princeton, NJ, 1987.

[21] R. W. Thomason. Une formule de Lefschetz en $K$-théorie équivariante algébrique. Duke Math. J., 68(3):447-462, 1992.

[22] Michela Varagnolo and Eric Vasserot. Double affine Hecke algebras and affine flag manifolds, I. In Affine flag manifolds and principal bundles, Trends Math., pages 233-289. Birkhäuser/Springer Basel AG, Basel, 2010.

[23] Marie-France Vignéras. Pro-p-Iwahori Hecke ring and supersingular $\overline{\mathbf{F}}_{p}$-representations. Math. Ann., $331(3): 523-556,2005$.

[24] Marie-France Vignéras. Algèbres de Hecke affines génériques. Represent. Theory, 10:1-20 (electronic), 2006.

[25] Marie-France Vignéras. The pro- $p$-Iwahori-Hecke algebra of a reductive $p$-adic group, II. Münster $J$. Math., 7:363-379, 2014.

Institute de Recherche Mathématique de Rennes, Université de Rennes 1, Campus de Beaulieu, 35042 Rennes, France

E-mail address: Tobias.Schmidt@univ-rennes1.fr 